\title{
Chapter 2 \\ Selection of Plants that Demonstrated Nitrate Removal Characteristics
}

In a joint project between two agencies in Singapore, the National Parks Board and the National University of Singapore-Singapore Delft Water Alliance, more than 30 plants species were screened and tested to select those that are suitable for application as vegetation in bioretention systems. The research project investigated the remediation capacity of the plants and their associated rhizosphere microbial communities. Of the numerous stormwater pollutants, the phytoremediation study focused on nitrogen.

The experiments were set up and conducted from September 2010 through June 2011 at a site in Singapore, with an average day time temperature of $32 \pm 1.87^{\circ} \mathrm{C}$ and night time temperature of $25 \pm 1.28^{\circ} \mathrm{C}$.

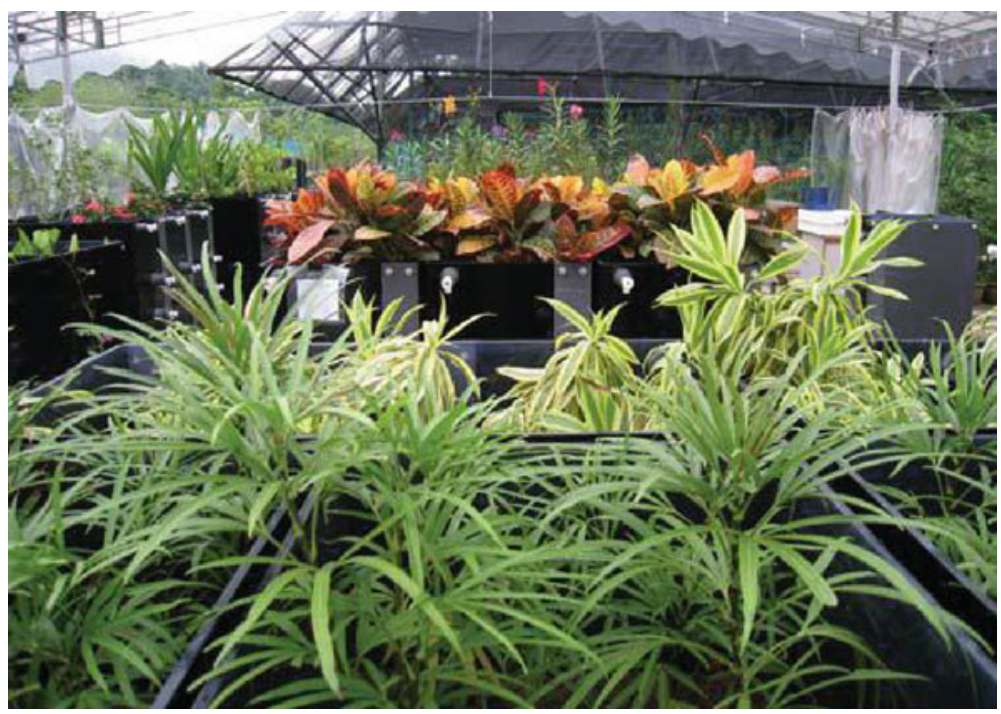

Experimental setup under a transparent pitched roof structure at a site in Singapore in 2010 


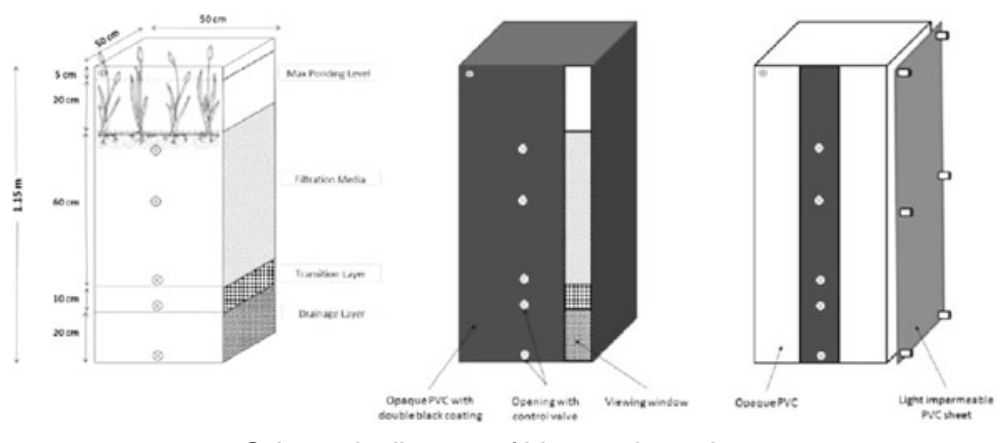

Schematic diagram of bioretention columns

The bioretention system comprised of three distinct layers: filtration layer, transition layer and drainage layer. The top filter media composed of $50 \%$ Singapore's Approved Soil Mix (ASM) and $50 \%$ medium to coarse sand. The transition media is made up of coarse sand and the bottom drainage media is made up of fine gravel. The filter media had a hydraulic conductivity of ca. $136 \mathrm{~mm} \mathrm{~h}^{-1}$, which was compliant with the 50-200 $\mathrm{mm} \mathrm{h}^{-1}$ range proposed in the bioretention design guidelines (PUB 2009).

\begin{tabular}{l|l|l|l}
\hline Layer & Substrate & Particle size $(\mathrm{mm})$ & Depth $(\mathrm{mm})$ \\
\hline Filter media & $\begin{array}{l}\text { Mixture of 50\% ASM } \\
\text { and 50\% medium to coarse sand }\end{array}$ & Varied & 600 \\
\hline Transition layer & Coarse sand & $0.7-1.0$ & 100 \\
\hline Drainage layer & Fine gravel & $1.0-5.0$ & 200 \\
\hline
\end{tabular}

To test the remediation capabilities of the plants for nutrients, irrigation water was chemically spiked to give a final concentration of $10 \mathrm{mg} \mathrm{L}^{-1}$ nitrate and $2 \mathrm{mg} \mathrm{L}^{-1}$ phosphate. These nutrient concentrations were above the levels commonly detected in urban stormwater runoffs, particularly in Singapore (Chua et al. 2009).

At the end of the experiment, the bioretention setups exhibited $100 \%$ efficiency in removing phosphate. However, phosphate was also completely removed in the unplanted control, indicating that the remediation of phosphate was primarily attributable to the bioretention substrate.

Eleven plant species demonstrated high efficiency in nitrate uptake, removing more than $85 \%$. The plant information of the 24 species with more than $50 \%$ nitrate uptake level is presented below. 

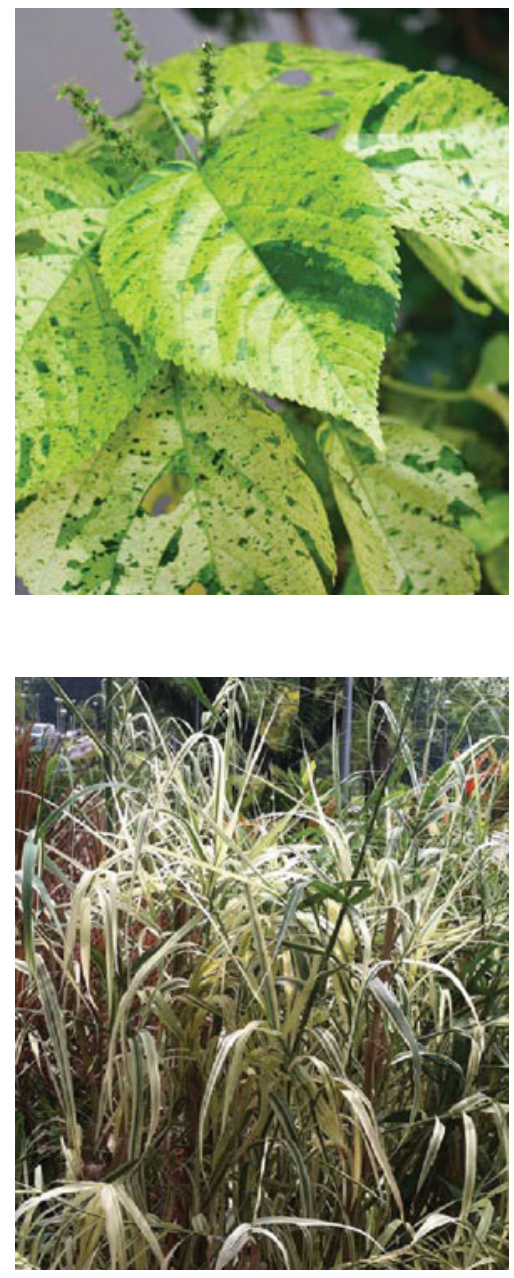

\section{Acalypha wilkesiana cultivar}

Family name: Euphorbiaceae

Common names: Copperleaf, Joseph's Coat

Plant growth form: Shrub

Maximum height: $3.6 \mathrm{~m}$

Maximum plant spread/crown width: $3 \mathrm{~m}$

Origin: South Pacific Islands

Landscape uses: Hedge; border; mass planting;

container or aboveground planter; screen; Accent

Desirable plant features: Ornamental foliage

Light preference: Full sun

Water preference: Moderate Water

Percentage of Nitrate Removal: $79 \%$

\section{Arundo donax var. versicolor}

Family name: Poaceae (Gramineae)

Common names: Carrizo, Variegated Giant Reed, Spanish Reed

Plant growth form: Shrub, Grass and Grass-like Plant

Maximum height: $6.1 \mathrm{~m}$

Origin: Asia

Landscape uses: Architectural accent/Screen

Desirable plant features: Ornamental Foliage

Light preference: Full Sun

Water preference: Moderate Water

Percentage of Nitrate Removal: $96 \%$ 


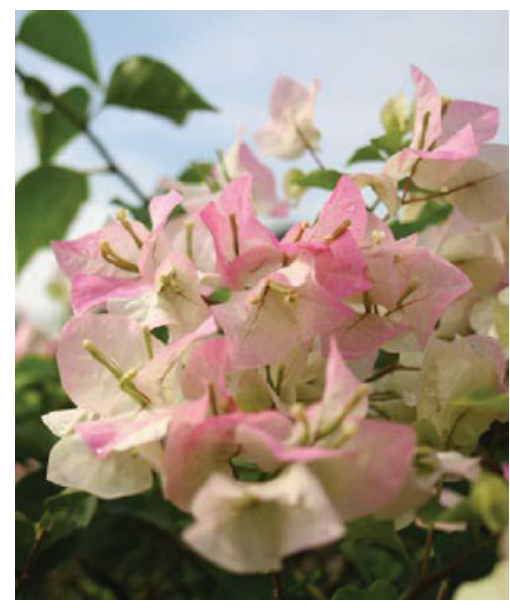

\section{Bougainvillea 'Sakura Variegata'}

Family name: Nyctaginaceae

Common names: Bougainvillea

Plant growth form: Scandent shrub

Origin : South America

Landscape uses: Shrubs, Bushes, Groundcover,

Trellis, Accent

Desirable plant features: Ornamental Flowers

Light preference: Full Sun

Water preference: Little Water

Percentage of Nitrate Removal: $96 \%$

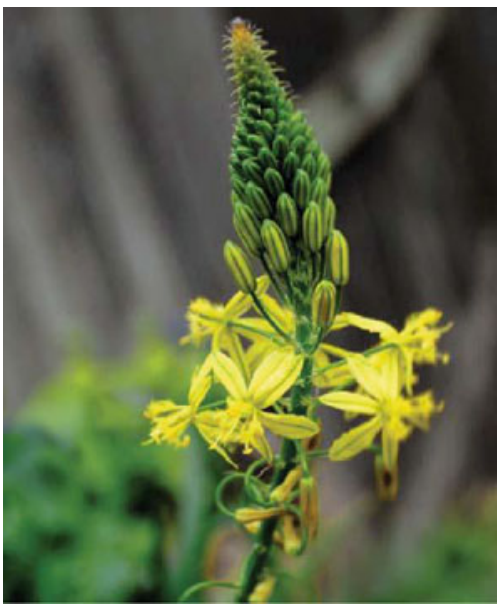

Bulbine frutescens (L.) Willd. 'Hallmark'

Family name: Asphodelaceae

Common names: Orange Bulbine, Orange-stalked

Bulbine

Plant growth form: Shrub (Herbaceous; Creeper)

Maximum height: $0.3-0.6 \mathrm{~m}$

Origin: Southern Africa

Landscape uses: Flowerbed/Border

Desirable plant features: Ornamental Flowers

Light preference: Full Sun

Water preference: Little Water

Percentage of Nitrate Removal: $68 \%$ 


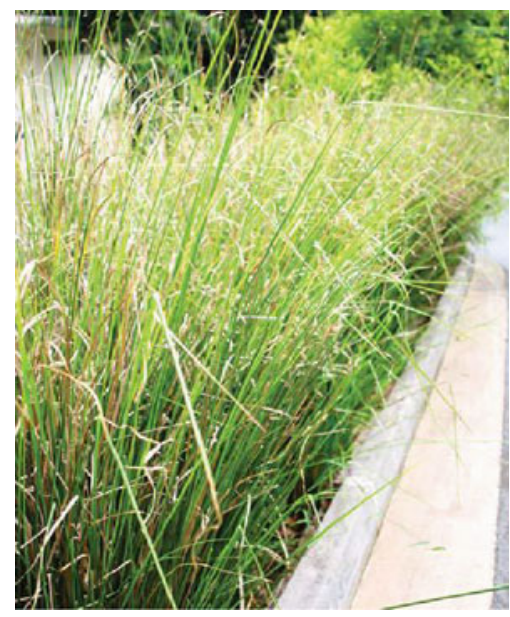

Chrysopogon zizanioides (L.) Roberty

Family name: Poaceae (Gramineae)

Common names: Vetiver Grass

Plant growth form: Shrub, Grass and Grass-like

Plant

Maximum height: $1.5 \mathrm{~m}$

Origin: Tropical India

Landscape uses: Hedge, Screening, Accent Plant,

Borders

Desirable plant features: Strong, deep roots for soil stabilisation

Light preference: Full Sun

Water preference: Moderate Water

Percentage of Nitrate Removal: $93 \%$

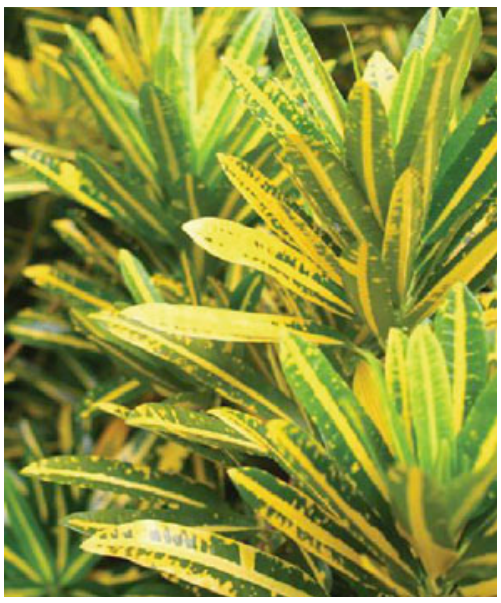

Codiaeum variegatum (L.) Rumph. ex A.Juss.

Synonyms: Croton pictus, Croton variegatus,

Codiaeum variegatum var. pictum

Family name: Euphorbiaceae

Common names: Croton, Garden Croton, Puding, Joseph's Coat, Variegated Croton, Variegated Laurel, Joseph's Coat, Puding, Codiaeum

Plant growth form: Shrub

Maximum height: $3 \mathrm{~m}$

Origin: South India, Ceylon and Malaya

Landscape uses: Indoor, General

Desirable plant features: Ornamental Foliage

Light preference: Full Sun

Water preference: Moderate Water

Percentage of Nitrate Removal: $67 \%$ 

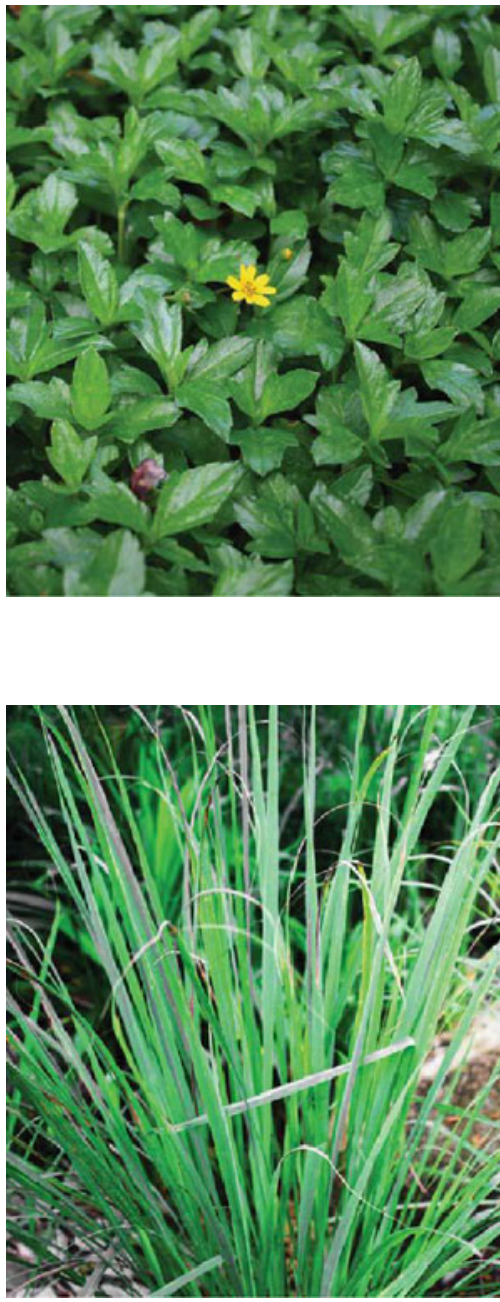

\section{Complaya trilobata}

Family name: Asteraceae (Compositae)

Synonyms: Wedelia trilobata, Wedelia carnosa,

Silphium trilobatum, Thelechitonia

trilobata, Sphagneticola trilobata (L.) Pruski

Common names: Yellow Creeping Daisy,

Singapore Daisy, Creeping Oxeye, Trailing Daisy, Rabbit's Paw

Plant growth form: Shrub

Maximum height: $0.15-0.70 \mathrm{~m}$

Maximum plant spread/crown width: $2 \mathrm{~m}$

Origin: Central America, South America

Desirable plant features: Ornamental flowers, ornamental foliage

Landscape uses: General, coastal, flowerbed/border, container planting

Light preference: Full sun, semi-shade

Water preference: Moderate water

Percentage of Nitrate Removal: $95 \%$

\section{Cymbopogon citratus (DC.) Stapf}

Synonyms: Andropogon citriodorum, Andropogon citratus

Family name: Poaceae (Gramineae)

Common names: Serai, Lemon Grass, West

Indian Lemon Grass, Oil Grass, Fever Grass,

Serai Makan, Sereh Makan

Plant growth form: Shrub, Grass and Grass-like

Plant

Maximum height: $1.2-1.8 \mathrm{~m}$

Maximum plant spread/crown width: $1.2 \mathrm{~m}$

Origin: Southeast Asia

Landscape uses: General, Flowerbed/Border,

Container Planting

Desirable plant features: Ornamental Foliage,

Fragrant (Flowers: Time Independent; Foliage)

Light preference: Full Sun

Water preference: Moderate Water

Percentage of Nitrate Removal: $95 \%$ 

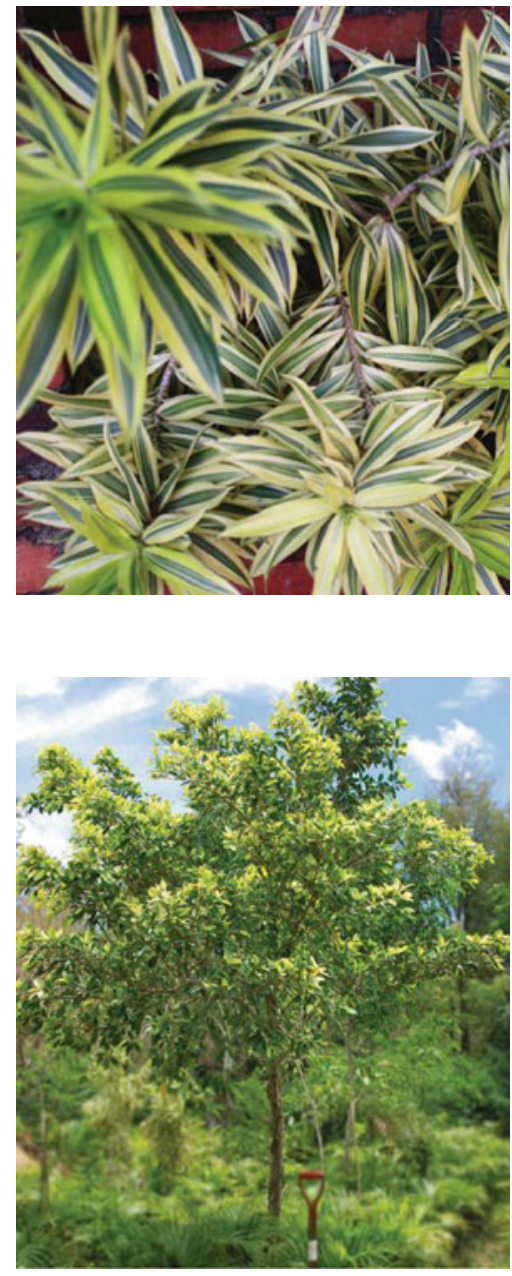

Dracaenaceae reflexa 'Song of India'

Family name: Dracaenaceae

Synonyms: Pleomele reflexa 'Variegata'

Common names: Song of India

Plant growth form: Shrub

Origin: South India, Ceylon

Desirable plant features: Ornamental Foliage

Landscape uses: General

Light preference: Full Sun, Semi-Shade

Water preference: Moderate Water

Percentage of Nitrate Removal: $64 \%$

\section{Ficus microcarpa 'Golden'}

Synonyms: Ficus nitida 'Golden'

Family name: Moraceae

Common names: Indian Laurel Fig

Plant growth form: Tree

Maximum height: $10 \mathrm{~m}$

Origin: India, Malaysia

Landscape uses: House plantings, Hedge, Roadside Plantings, Bonsai

Desirable plant features: Ornamental Foliage

Light preference: Full Sun

Water preference: Moderate Water

Percentage of Nitrate Removal: $68 \%$ 

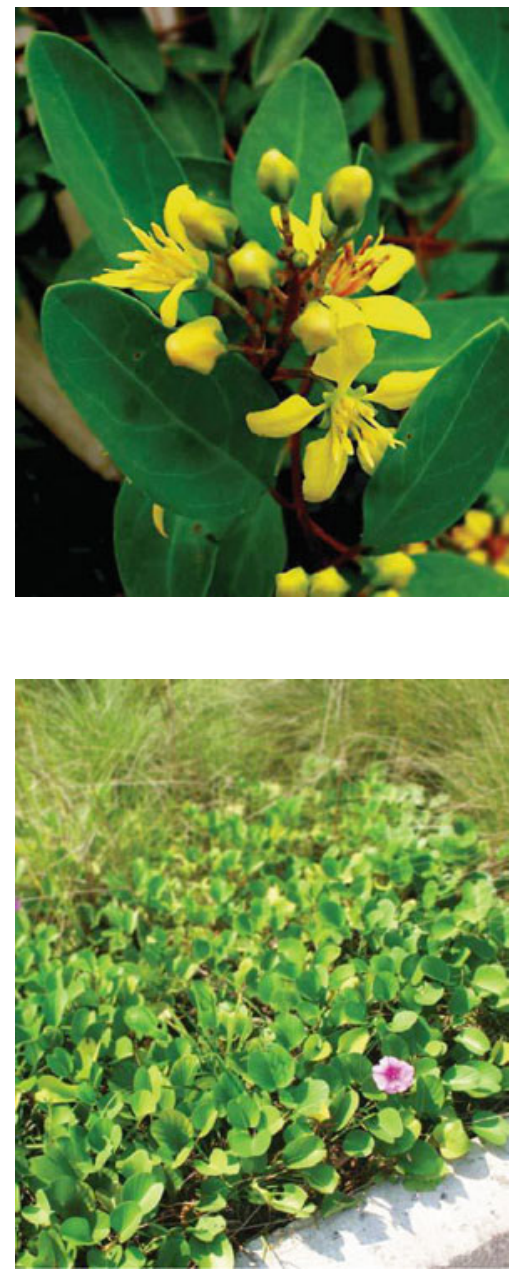

Galphimia glauca Cav.

Synonyms: Thryallis glauca

Family name: Malpighiaceae

Common names: Shower of Gold, Rain of Gold

Plant growth form: Shrub (Woody)

Maximum height: $1.0 \mathrm{~m}$

Origin: Mexico to Guatemala

Landscape uses: Hedge/Screening, Groundcover,

Container planting

Desirable plant features: Ornamental Flowers

Light preference: Full Sun

Water preference: Little Water

Percentage of Nitrate Removal: $65 \%$

\section{Ipomoea pes-caprae}

Family name: Convolvulaceae

Synonyms: Ipomoea pes-caprae ssp. Brasiliensis, Ipomoea biloba

Common names: Beach Morning Glory, Railroad

Vine, Sea Morning Glory, Tapak Kuda, Goat's

Foot

Plant growth form: Groundcover

Origin: Native to Singapore

Desirable plant features: Ornamental flowers,

ornamental foliage

Landscape uses: Coastal

Light preference: Full sun

Water preference: Lots of water

Flower Colour(s): Purple

Percentage of Nitrate Removal: $95 \%$ 

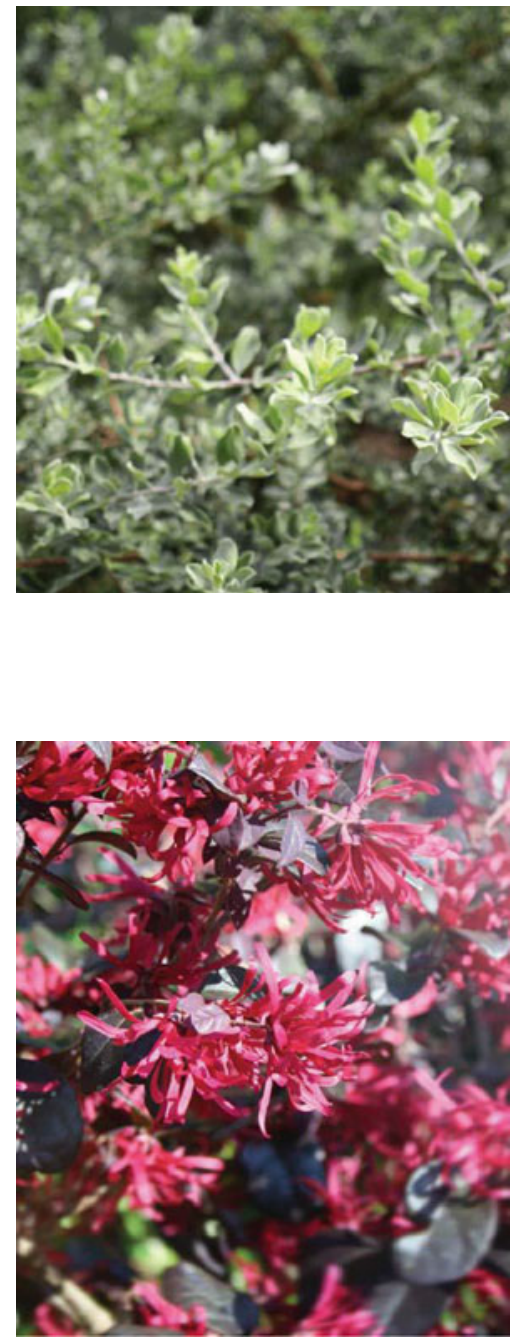

\section{Leucophyllum frutescens (Berland.) I.}

M. Johnst.

Family name: Scrophulariaceae

Common names: Barometer Bush, Ash Plant, Cenizo, Texas Ranger, Texas Silver Leaf, Purple Sage

Plant growth form: Shrub (Woody)

Maximum height: $1.2-2.4 \mathrm{~m}$

Maximum plant spread/crown width: $1.5 \mathrm{~m}$ Origin: Rio Grande Valley (Texas, New Mexico), northern Mexico

Landscape uses: General, Coastal, Hedge/Screening, Flowerbed/Border, Container Planting Desirable plant features: Ornamental Flowers, Ornamental Foliage, Ornamental Stems, Fragrant (Flowers: Day; [Remarks]: Peppery/spicy scent, reminiscent of carnations) Light preference: Full Sun Water preference: Little Water

Percentage of Nitrate Removal: $87 \%$

\section{Loropetalum chinense (R. Br.) Oliv.}

Family name: Hamamelidaceae

Common names: Chinese Loropetalum Plant growth form: Shrub

Maximum height: $1.5 \mathrm{~m}$

Origin: China

Landscape uses: Hedge, Garden Plant

Desirable plant features: Ornamental Flowers,

Ornamental Foliage

Light preference: Full Sun

Water preference: Moderate Water

Percentage of Nitrate Removal: $71 \%$ 


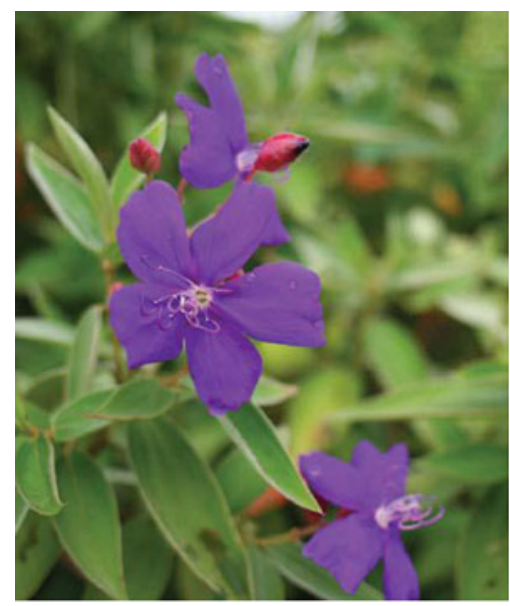

\section{Melastoma malabathricum L.}

Family name: Melastomataceae

Common names: Common Sendudok, Singapore

Rhododendron, Indian Rhododendron, Sesenduk, Malabar Gooseberry, Straits Rhododendron,

Sendudok, Senduduk

Plant growth form: Shrub (Herbaceous)

Maximum height: 1-3 m

Origin: Mauritius, Seychelles, Taiwan, Indian subcontinent, Indo-China, Malesia, Australia Landscape uses: Invasive/Potentially Invasive, [Remarks] (Considered weedy. May form thick thickets)

Desirable plant features: Ornamental Flowers

Light preference: Full Sun, Semi-Shade

Water preference: Moderate Water

Percentage of Nitrate Removal: $68 \%$

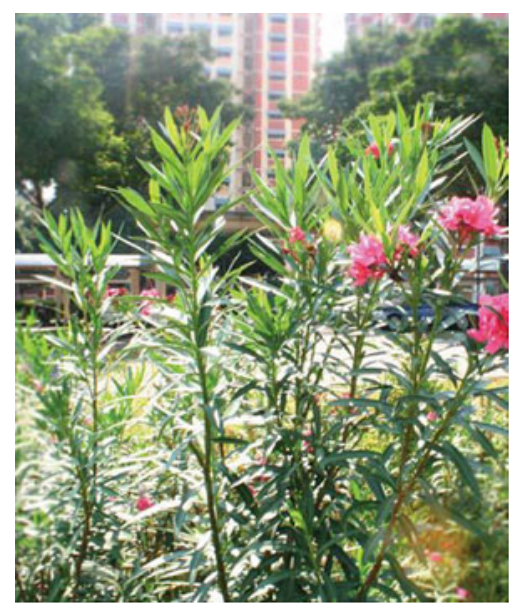

\section{Nerium oleander 'Pink'}

Family name: Apocynaceae

Common names: Oleander

Plant growth form: Shrub

Maximum height: 6-7 m

Origin: Unknown, probably South-west Asia Landscape uses: Interiorscape/ Indoor Plant,

Hedges, Borders

Desirable plant features: Ornamental Flowers, Fragrant

Light preference: Full Sun

Water preference: Moderate Water

Percentage of Nitrate Removal: $88 \%$ 

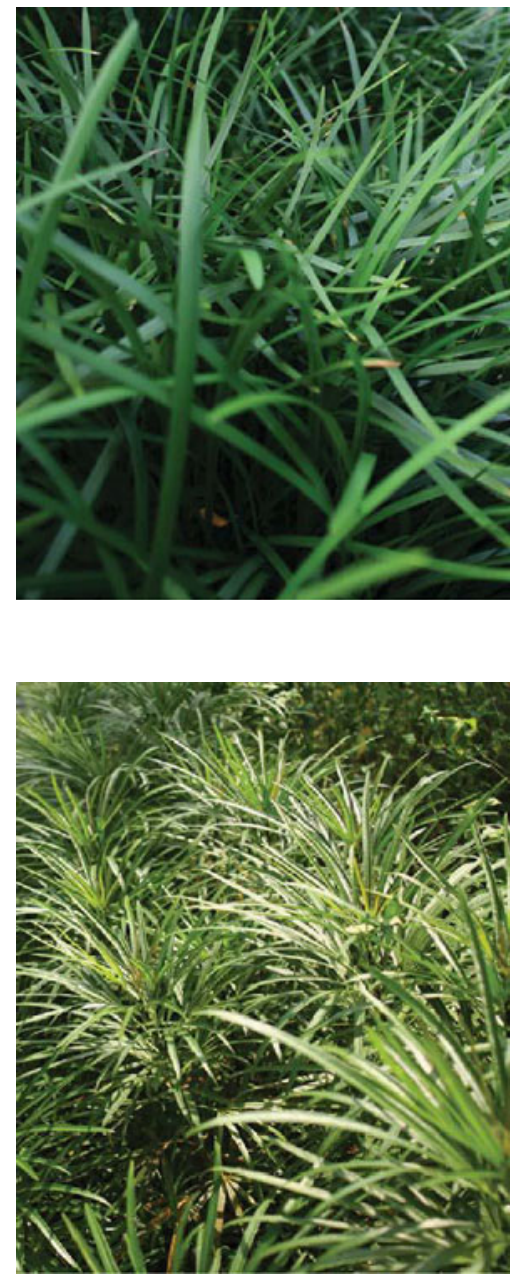

\section{Ophiopogon jaburan}

Family name: Convallariaceae

Common names: Lilyturf, Mondo-grass

Plant growth form: Groundcover

Origin: Japan

Landscape uses: Ornamental plants in gardening, houseplants

Desirable plant features: Ornamental foliage

Light preference: Full sun, semi-shade

Water preference: Moderate water

Percentage of Nitrate Removal: $77 \%$

\section{Osmoxylon lineare (Merr.) Philipson}

Synonyms: Boerlagiodendron lineare

Family name: Araliaceae

Common names: Green Aralia, Miagos Bush

Plant growth form: Shrub (Herbaceous)

Maximum height: $3 \mathrm{~m}$

Origin: South-east Asia

Landscape uses: This species is used in Japanese-

style gardens for its fine leaf texture

Desirable plant features: Ornamental Foliage

Light preference: Full Sun

Water preference: Moderate Water

Percentage of Nitrate Removal: $66 \%$ 

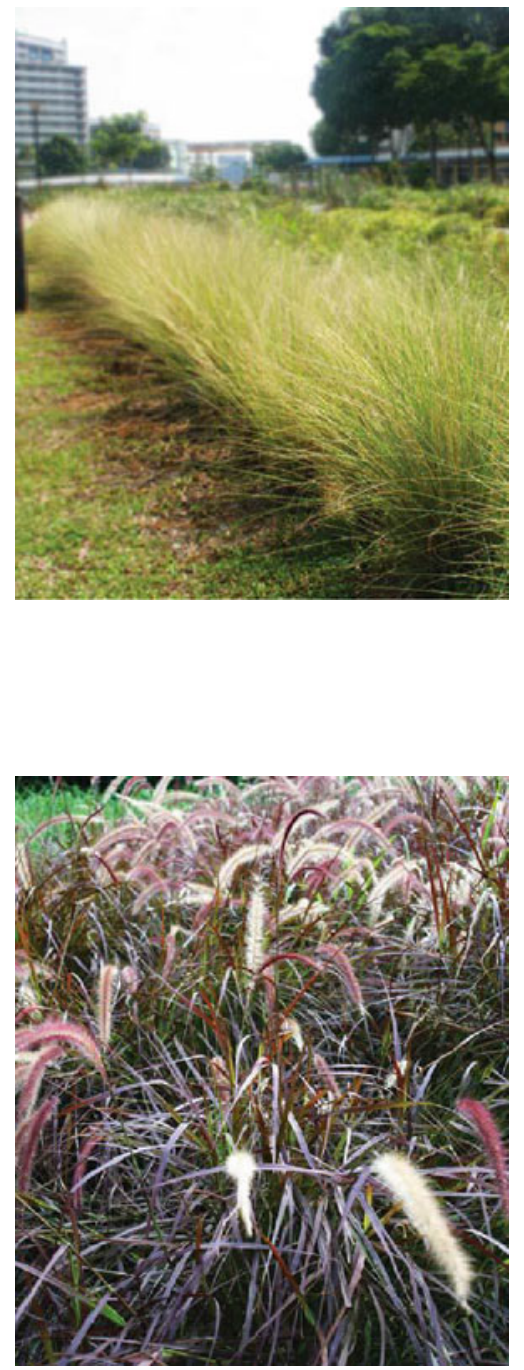

Pennisetum alopecuroides (L.) Spreng.

Synonyms: Panicum alopecuroides, Alopecurus hordeiformis, Pennisetum

hordeiforme, Pennisetum compressum,

Pennisetum japonicum

Family name: Poaceae (Gramineae)

Common names: Chinese Fountain Grass, Swamp

Foxtail Grass, Swamp Foxtail, Chinese

Pennisetum

Plant growth form: Shrub (Herbaceous), Grass and Grass-like Plant

Maximum height: $0.6-1.5 \mathrm{~m}$

Maximum plant spread/crown width: 0.5-0.6 m

Origin: India, China, Japan, Myanmar,

Philippines, Indonesia, Australia

Landscape uses: General, Coastal, Flowerbed/

Border, Container Planting

Desirable plant features: Ornamental Flowers

Light preference: Full Sun

Water preference: Lots of Water, Moderate Water

Percentage of Nitrate Removal: $93 \%$

\section{Pennisetum $x$ advena 'Rubrum'}

Synonyms: Pennisetum setaceum 'Cupreum', Pennisetum setaceum 'Rubrum'

Family name: Poaceae (Gramineae)

Common names: Purple Fountain Grass, Red

Fountain Grass, Rose Fountain Grass

Plant growth form: Shrub (Herbaceous; Creeper)

Maximum height: $1.2-1.5 \mathrm{~m}$

Maximum plant spread/crown width: $0.5-1.2 \mathrm{~m}$

Origin: Central Africa

Landscape uses: General, Coastal, Green Roof,

Vertical Greenery/Green

Wall, Flowerbed/Border, Focal Plant, Container

Planting

Desirable plant features: Ornamental Flowers,

Ornamental Foliage

Light preference: Full Sun

Water preference: Moderate Water

Percentage of Nitrate Removal: $70 \%$ 

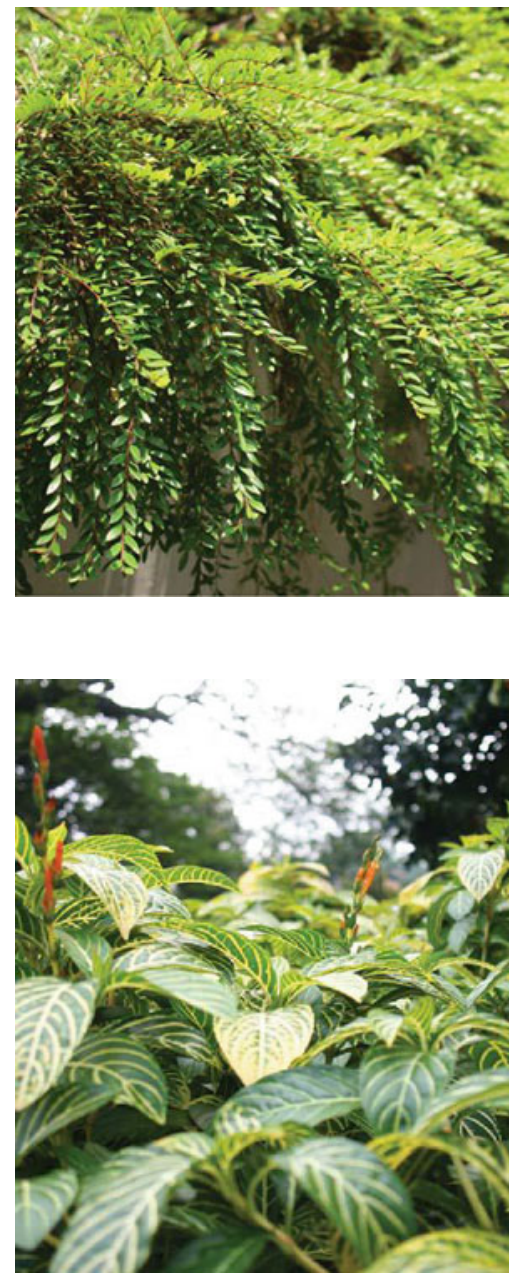

Phyllanthus myrtifolius Müll.Arg.

Family name: Euphorbiaceae

Common names: Ceylon Myrtle, Mousetail Plant

Plant growth form: Shrub

Maximum height: $0.5 \mathrm{~m}$

Origin: India, Sri Lanka

Landscape uses: Flowerbed/Border, Bonsai

Desirable plant features: Ornamental Foliage

Light preference: Full Sun

Water preference: Moderate Water

Percentage of Nitrate Removal: $55 \%$

\section{Sanchezia oblonga Ruiz \& Pav.}

Synonyms: Sanchezia speciosa, Sanchezia nobilis Family name: Acanthaceae

Common names: Zebra Plant, Yellow Sanchezia, Shrubby Whitevein, Gold Vein Plant

Plant growth form: Shrub (Herbaceous)

Maximum height: $1-3 \mathrm{~m}$

Origin: Eucador, Northeastern Peru

Landscape uses: Hedge/Screening, Flowerbed/

Border, Interiorscape/

Indoor Plant, Container Planting

Desirable plant features: Ornamental Flowers,

Ornamental Foliage

Light preference: Full Sun, Semi-Shade

Water preference: Moderate Water

Percentage of Nitrate Removal: 87 \% 


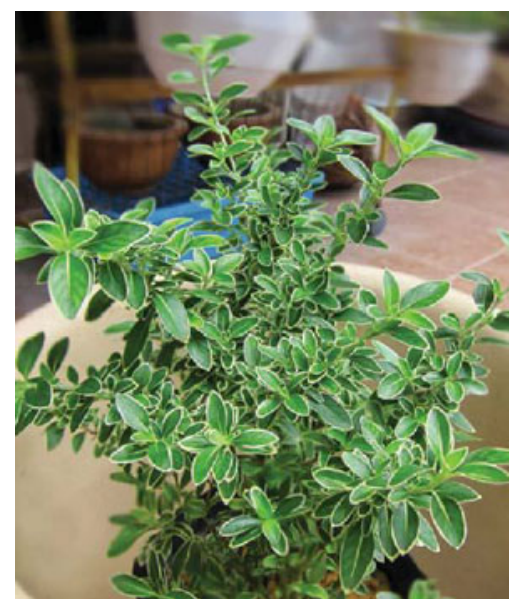

Serissa japonica (Thunb.) Thunb.

Synonyms: Serissa foetida

Family name: Rubiaceae

Common names: Japanese Serissa, Snowrose,

Tree of a Thousand Stars, Japanese Boxthorn

Plant growth form: Shrub (Woody)

Maximum height: $0.45-0.60 \mathrm{~m}$

Origin: India, China, Japan

Landscape uses: General, Coastal, Container

Planting, Bonsai

Desirable plant features: Ornamental Flowers,

Ornamental Foliage, Ornamental Stems, Fragrant

(Flowers: Time Independent; Foliage; Stems)

Light preference: Full Sun, Semi-Shade

Water preference: Moderate Water

Percentage of Nitrate Removal: $71 \%$

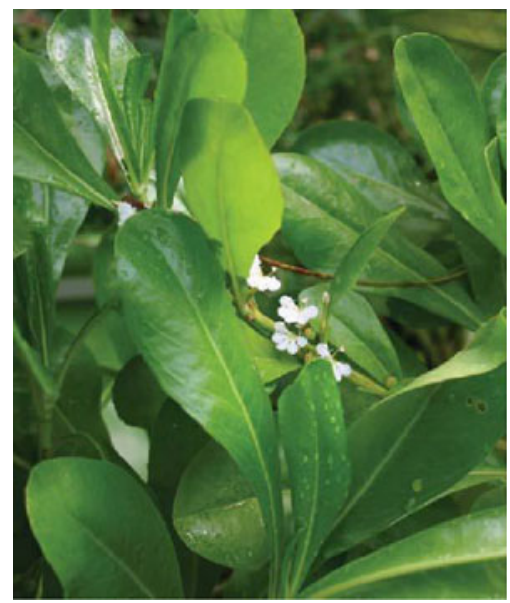

Scaevola taccada (Gaertn.) Roxb.

Synonyms: Scaevola sericea, Lobelia taccada, Scaevola frutescens

Family name: Goodeniaceae

Common names: Ambung-ambung, Merambung,

Pelampung, Sea Lettuce, Sea Lettuce Tree

Plant growth form: Tree, Shrub (Herbaceous;

Creeper)

Maximum height: $10 \mathrm{~m}$

Origin: S.E.A. to tropical Australia, Micronesia,

Melanesia, Hawaii and

Madagascar

Landscape uses: Coastal

Desirable plant features: Ornamental Foliage

Light preference: Full Sun

Water preference: Moderate Water

Percentage of Nitrate Removal: $88 \%$

Open Access This chapter is distributed under the terms of the Creative Commons Attribution Noncommercial License, which permits any noncommercial use, distribution, and reproduction in any medium, provided the original author(s) and source are credited. 